\title{
Rabi oscillations under ultrafast excitation of graphene
}

\author{
P.N. Romanets and F.T. Vaskd* \\ Institute of Semiconductor Physics, NAS of Ukraine, Pr. Nauky 41, Kiev, 03028, Ukraine
}

(Dated: October 30, 2018)

\begin{abstract}
We study coherent nonlinear dynamics of carriers under ultrafast interband excitation of an intrinsic graphene. The Rabi oscillations of response appear with increasing of pumping intensity. The photoexcited distribution is calculated versus time and energy taking into account the effects of energy relaxation and dephasing. Spectral and temporal dependencies of the response on a probe radiation (transmission and reflection coefficients) are considered for different pumping intensities and the Rabi oscillations versus time and intensity are analyzed.
\end{abstract}

PACS numbers: 78.47.jh, 78.67.Wj

The Rabi oscillations of coherent response under ultrafast excitation of two-level atomic systems were studied during last decades. 11 Similar phenomena in bulk semiconductors and quantum wells have also been observed, see [2, 3] and reviews. [4, 5] Recently, the properties of graphene under ultrafast interband excitation were investigated, and most attention has been concentrated on relaxation dynamics for the case of the linear excitation regime of epitaxial and exfoliated graphene, see [6] and 7] respectively. It was found that the relaxation times of photoexcited electron-hole pairs due to the optical phonon emission are about 0.1 ps [6, 7], so that an investigation of coherent dynamics is possible during the femtosecond time scales when the regime of Rabi oscillations can be realized with increasing of pumping intensity. To the best of our knowledge, neither experimental nor theoretical treatment of the nonlinear coherent response for the Rabi oscillations regime in graphene is not performed yet. Due to the gapless and massless energy spectrum with a neutrinolike dispersion law $\pm v_{W} p\left(v_{W} \simeq 10^{8} \mathrm{~cm} / \mathrm{s}\right.$ is the characteristic velocity for the Weyl-Wallace model [8]), such a case should be essentially different from the above mentioned cases. [2 5]

In this paper, we consider the nonlinear coherent process of carrier photoexcitation in an intrinsic graphene under the slow-envelope condition, $\Omega \tau_{p} \gg 1$ where $\Omega$ is the frequency of light and $\tau_{p}$ is the duration of excitation. Eliminating the nondiagonal components of density matrix, which are responsible for the high-frequency $[\propto \exp (-i \Omega t)]$ oscillations of polarization, we describe such a process by the same distribution functions for electrons and holes, $f_{p t}$ because of the electron-hole symmetry. The kinetic equation for $f_{p t}$ takes form

$$
\frac{d f_{p t}}{d t}=G\left(f_{p} \mid t\right)+J_{0}\left(f_{t} \mid p\right)
$$

where $G\left(f_{p} \mid t\right)$ is the interband generation rate and $J_{0}\left(f_{t} \mid p\right)$ is the collision integral. The general consideration of Eq. (1) can be found in [9] and the evaluation of the generation rate for graphene is performed in Ref. 10. Further, we solve this equation and analyze the spectral and temporal dependencies of the transmission and reflection coefficients for a probe radiation at different pumping levels.

The coherent interband photoexcitation caused by the in-plane electric field $w_{t} \mathbf{E} \exp (-i \Omega t)+$ c.c. with the envelope form-factor $w_{t}$ is described by the generation rate in Eq. (1) [10]

$$
\begin{array}{r}
G\left(f_{p} \mid t\right)=\left(\frac{e E v_{W}}{\hbar \Omega}\right)^{2} w_{t} \int_{-\infty}^{0} d t^{\prime} w_{t+t^{\prime}} e^{t^{\prime} / \tau_{d}} \\
\times \cos \left[\left(\frac{2 v_{W} p}{\hbar}-\Omega\right) t^{\prime}\right]\left(1-2 f_{p t+t^{\prime}}\right) .
\end{array}
$$

Here we neglect the multi-photon interband transitions under the condition $\left(e E v_{W} / \hbar \Omega^{2}\right)^{2} \ll 1$ and the dephasing relaxation time $\tau_{d}$ is introduced phenomenologically. The non-local factor $\left(1-2 f_{p t+t^{\prime}}\right)$ describes the Pauli blocking and the temporal memory effect which is responsible for the Rabi oscillations. Since the cascade emission of optical phonons dominates in the energy relaxation, [6, 7, 11] Eq. (1) involves the collision integral

$$
J_{0}\left(f_{t} \mid p\right)=\nu_{p+p_{0}}\left(1-f_{p t}\right) f_{p+p_{0} t}-\nu_{p-p_{0}}\left(1-f_{p-p_{0} t}\right) f_{p t},
$$

where $p_{0}=\hbar \omega_{0} / v_{W}$ is the characteristic momentum, $\hbar \omega_{0}$ is the optical phonon energy, and $\nu_{p}=v_{0} p / \hbar$ is the relaxation rate for the spontaneous emission of optical phonons. Under the condition $\nu_{p_{0}} \tau_{p} \ll 1$ we neglect interband

*Electronic address: ftvasko@yahoo.com 
transitions due to optical phonon emission. We also restrict ourselves by the model with a single phonon of energy $\hbar \omega_{0} \simeq 0.2 \mathrm{eV}$ with the efficiency of coupling determined by the characteristic velocity $v_{0} \simeq 10^{6} \mathrm{~cm} / \mathrm{s}$. These parameters are in agreement with the results of calculations of the electron-phonon interaction [12] and with the measurements of relaxation dynamics. [6, 7] The problem given by Eqs. (1) - (3) is solved below with the initial condition $f_{p t \rightarrow-\infty}=0$ which is correspondent to the undoped graphene.

The analytical solution of the problem formulated can be found for the collisionless case, $\tau_{d} \rightarrow \infty$ and $v_{0} \rightarrow 0$, under the resonant condition $p \rightarrow \hbar \Omega / 2 v_{W} \equiv p_{\Omega}$, when the oscillating factor is absent in the integral generation rate (2). For such a case, the integro-differential Eq. (1) can be transformed into the second order differential equation and the solution takes form

$$
f_{p=p_{\Omega}, t}=\frac{1}{2}\left[1-\cos \left(\sqrt{2 I_{e x}} \int_{-\infty}^{t} \frac{d t^{\prime}}{\tau_{p}} w_{t^{\prime}}\right)\right] .
$$

Here we introduced the dimensionless intensity, $I_{e x}=\left(e E \tau_{p} v_{W} / \hbar \Omega\right)^{2}$, so that the Rabi oscillations of the resonant distribution with time and with field strength $\left(\sqrt{2 I_{e x}} \propto E\right)$ takes place. At $t \rightarrow \infty$ one obtains the resonant distribution $f_{p=p_{\Omega}, t \rightarrow \infty}=\left(1-\cos \mathcal{A}_{e x}\right) / 2$ which is determined by the dimensionless area of the incident pulse $\mathcal{A}_{e x}=$ $\sqrt{2 I_{e x}} \int_{-\infty}^{\infty} d t w_{t} / \tau_{p}$

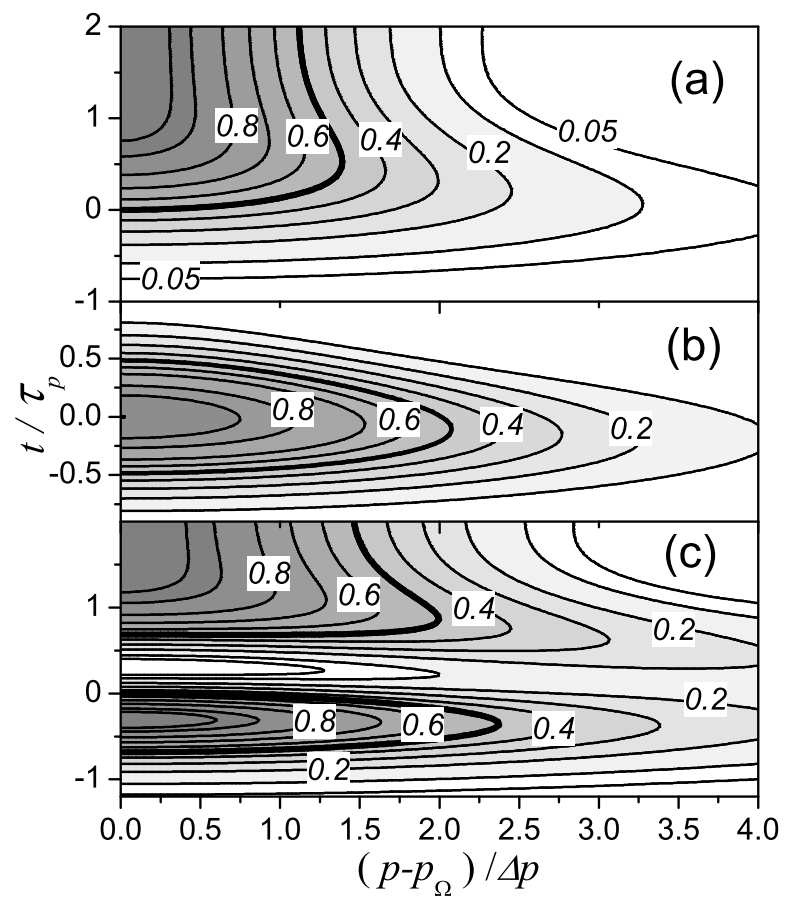

FIG. 1: Contour plots of photoexcited distribution $f_{p t}$ versus dimensionless momentum and time, $\left(p-p_{\Omega}\right) / \Delta p$ and $t / \tau_{p}$ for the collisionless regime at pumping levels corresponding to $\mathcal{A}_{\text {ex }}=\pi$ (a), $2 \pi$ (b) and $3 \pi$ (c).

The collisionless case at $p \neq p_{\Omega}$ is described by the reduced equation $(1) d f_{p t} / d t=G\left(f_{p} \mid t\right)$. The numerical solution of this equation is obtained here with the use of the finite difference method [13] and the Gaussian form-factor $w_{t}=\sqrt[4]{2 / \pi} \exp \left[-\left(t / \tau_{p}\right)^{2}\right]$. [14] In Fig. 1 we plot $f_{p t}$ versus dimensionless time, $t / \tau_{p}$, and momentum $\left(p-p_{\Omega}\right) / \Delta p$ which is centered at $p_{\Omega}$. Here $\Delta p=\hbar /\left(v_{W} \tau_{p}\right)$ determines a width of photoexcited distribution at $t \geq 2 \tau_{p}$ while a width of distribution at $t \simeq 0$ increases with pumping intensity, as it is shown in Figs. 1 a-c. As $I_{e x}$ increases, a temporal Rabi oscillations at $p \neq p_{\Omega}$ are similar to the transient evolution described by Eq. (4).

We turn now to consideration of the problem (1) - (3) taking into account the dephasing and energy relaxation processes, when $\tau_{d}$ and $v_{0}$ are finite. In the calculations below we use the pumping frequency $\hbar \Omega=1.5 \mathrm{eV}$ and the dephasing time $\tau_{d} \simeq \nu_{p_{\Omega}}^{-1}=85 \mathrm{fs}$, which is correspondent to the process of spontaneous emission of optical phonons. Since the cascade emission of dispersionless optical phonons, described by Eq. (3), a multipeak structure of $f_{p t}$ with maxima centered around $p_{\Omega}-k p_{0}(k=0,1, \ldots)$ is realized. The only first phonon repetition is essential during the photoexcitation process, when $t \leq 2 \tau_{p}$, see Figs. $2 \mathrm{a}$ and $2 \mathrm{~b}$ where the contour plots of the photoexcited distributions $f_{p t}$ at the pumping level $\mathcal{A}_{e x}=3 \pi$ are shown for the pulse durations $\tau_{p}=30 \mathrm{fs}$ and 80 fs. One can see that the 


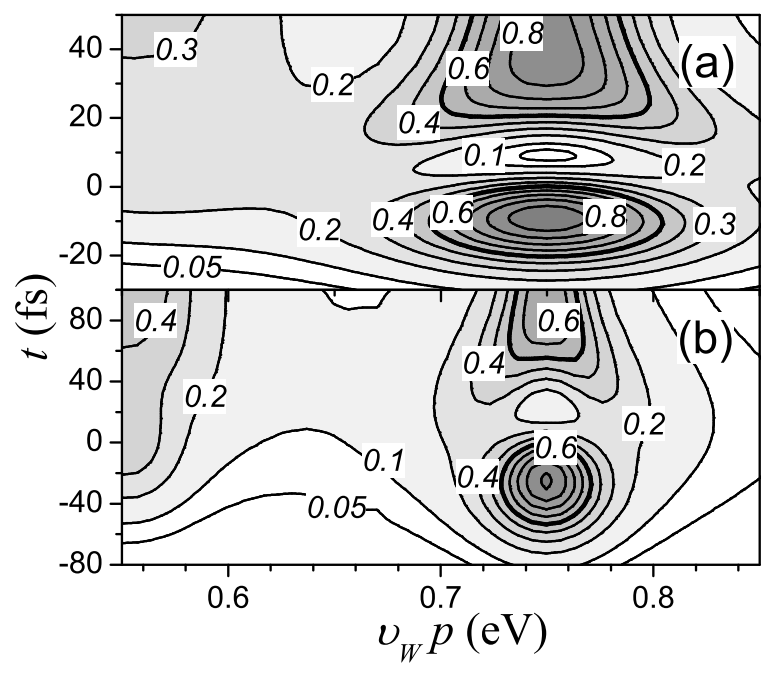

FIG. 2: Contour plots of photoexcited distributions $f_{p t}$ versus energy $v_{W} p$ and time for pulse durations $\tau_{p}=30$ fs (a) and 80 fs (b) at pumping level corresponding to $\mathcal{A}_{\text {ex }}=3 \pi$.

photoexcited distribution appears to be narrower with increasing of $\tau_{p}$ and that the Rabi oscillations are visible at $\tau_{d} \sim \tau_{p}$ (Fig. 2b).

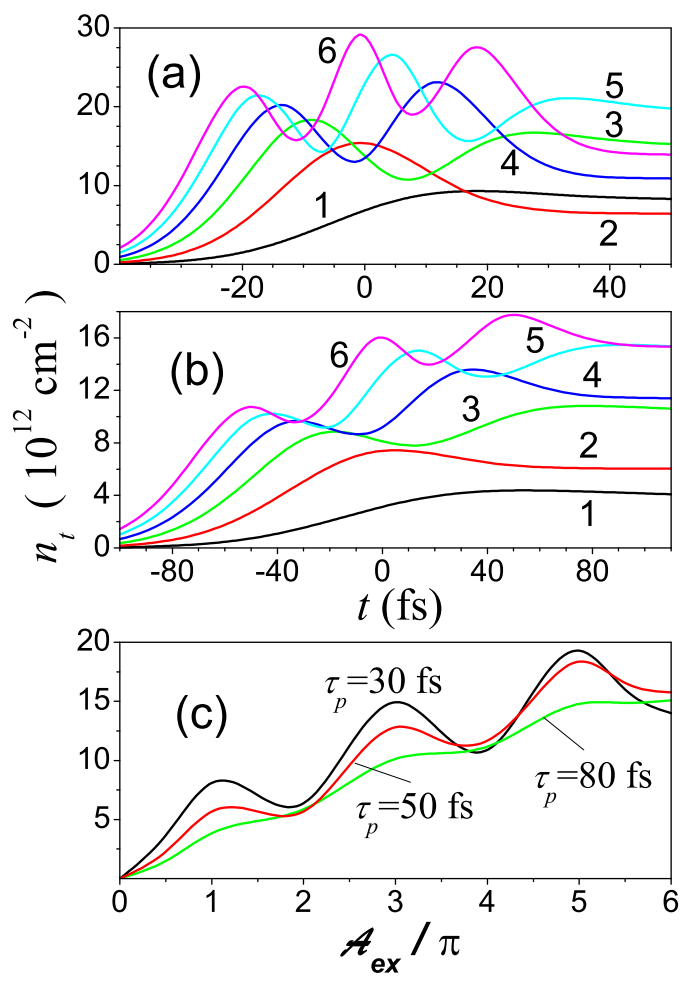

FIG. 3: (Color online) Transient evolution of concentration of photoexcited carriers for pulse durations $\tau_{p}=30$ fs (a) and 80 fs (b) at pumping levels corresponding to $\mathcal{A}_{e x}=\pi(1), 2 \pi(2), 3 \pi(3), 4 \pi(4), 5 \pi(5)$, and $6 \pi(6)$. (c) Photoexcited concentration versus $\mathcal{A}_{\text {ex }}$ at $t \gg \tau_{p}$ for different $\tau_{p}$ (marked).

Using the distribution $f_{p t}$ obtained, we calculate here the concentration of photoexcited carriers

$$
n_{t}=\frac{2}{\pi \hbar^{2}} \int_{0}^{\infty} d p p f_{p t}
$$


versus time and versus pumping level at $t \gg \tau_{p}$. As one can see from Figs. 3a and 3b, the amplitudes of temporal oscillations decreases with increasing of $\tau_{p}$ but the oscillations remain visible at $\tau_{p} \sim \tau_{d}$. The oscillatory behavior of $n_{t \rightarrow \infty}$ versus pumping level (which is proportional to $\mathcal{A}_{e x}^{2}$ ) appears to be suppressed at $\tau_{p} \sim \tau_{d}$ as it is shown in Fig. 3c. The condition $\mathcal{A}_{e x}=\pi$ corresponds to the pulse energies $\sim 15 \mathrm{~nJ}$ or $\sim 5.6 \mathrm{~nJ}$ for $\tau_{p}=30$ fs or 80 fs and for the sport area $\sim 10^{-4} \mathrm{~cm}^{2}$ (note, that the pulse energy $\propto \tau_{p}^{-1}$ and $\propto n^{2}$, if $\mathcal{A}_{e x}=n \pi$ ).

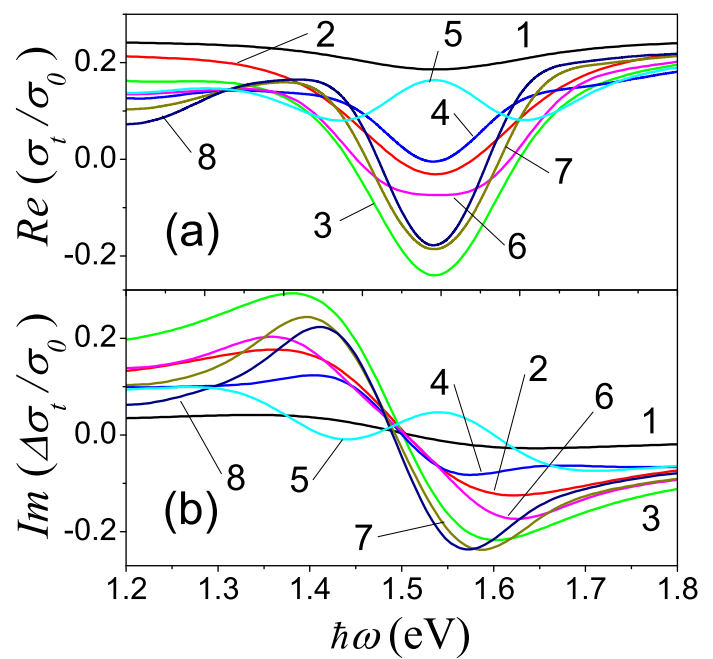

FIG. 4: (Color online) Spectral dependencies of the dynamic conductivity, Re $\sigma_{\omega t}$ (a) and $\operatorname{Im} \Delta \sigma_{\omega t}$ (b), around the pumping energy $1.5 \mathrm{eV}$ for delay times between -30 and $40 \mathrm{fs}$ with step $10 \mathrm{fs}$ (marked by 1 - 8) at pumping level corresponding to $\mathcal{A}_{e x}=3 \pi$ and pulse duration $\tau_{p}=30$ fs.

The transient response on a probe radiation $\propto \exp (-i \omega t)$ is determined by the dynamic conductivity $\sigma_{\omega t}$ which was evaluated in [9, 15] for the collisionless case $\omega \tau_{d} \gg 1$, when the parametric dependency on time takes place. The real and imaginary parts of $\sigma_{\omega t}$ are given by

$$
\begin{gathered}
\operatorname{Re} \sigma_{\omega t}=\frac{e^{2}}{4 \hbar}\left(1-2 f_{p_{\omega}, t}\right), \\
\operatorname{Im} \sigma_{\omega t}=\bar{\sigma}_{\omega}-\frac{e^{2}}{\pi \hbar} \mathcal{P} \int_{0}^{\infty} \frac{d y y^{2}}{1-y^{2}} f_{p_{\omega} y, t},
\end{gathered}
$$

where $\mathcal{P}$ means the principal value of integral and we introduced the time-independent contribution $\bar{\sigma}_{\omega}$ described the undoped graphene in the absence of photoexcitation (below we use the phenomenological expression for $\bar{\sigma}_{\omega}$ introduced in [16]). The negative absorption condition $\operatorname{Re} \sigma_{\omega t}<0$ takes place if $f_{p_{\omega}, t}>1 / 2$; these regions were marked by thick curves in Figs. 1 and 2. The peak shape of $\operatorname{Re} \sigma_{\omega t}$ centered at $\omega=\Omega$ leads to a visible spectral dispersion of Im $\sigma_{\omega t}$ at $\omega<\Omega$ and $\omega>\Omega$. [16]

The reflection and transmission coefficients, $R_{\omega t}$ and $T_{\omega t}$, are written through the dynamic conductivity as follows:

$$
\begin{aligned}
R_{\omega t} & =\left|\frac{1-\sqrt{\epsilon}-4 \pi \sigma_{\omega t} / c}{1+\sqrt{\epsilon}+4 \pi \sigma_{\omega t} / c}\right|^{2} \\
T_{\omega t} & =\frac{4 \sqrt{\epsilon}}{\left|1+\sqrt{\epsilon}+4 \pi \sigma_{\omega t} / c\right|^{2}} .
\end{aligned}
$$

Here $\epsilon$ is the dielectric permittivity of a thick substrate and the geometry of normal propagation of radiation was considered. The contour plots of $R_{\omega t}$ and $T_{\omega t}$ versus $t$ and $\omega$ are shown in Fig. 5 at pumping level corresponding to $\mathcal{A}_{e x}=3 \pi$ for $\tau_{p}=30 \mathrm{fs}$ and $80 \mathrm{fs}$. Temporal oscillations of response correlate with the evolution of $\sigma_{\omega t}($ Fig. 3 ) due to oscillations of distribution shown in Fig. 2. Spectral width of Rabi oscillations appears to be broader for $\tau_{p}=30$ fs (c.f. Figs. 5a, 5b and 5c, 5d with Figs. 2a and 2b). The oscillatory behaviors of the reflection and transmission coefficients at the pumping frequency $(\omega=\Omega), R_{\Omega t}$ and $T_{\Omega t}$, versus pumping level for the delay times $\tau_{p}, 2 \tau_{p}$, and $3 \tau_{p}$ are shown in Figs. $6 \mathrm{a}$ and $6 \mathrm{~b}$, respectively. One can see a few-percent modulation of response at $t \geq \tau_{p}$ versus 

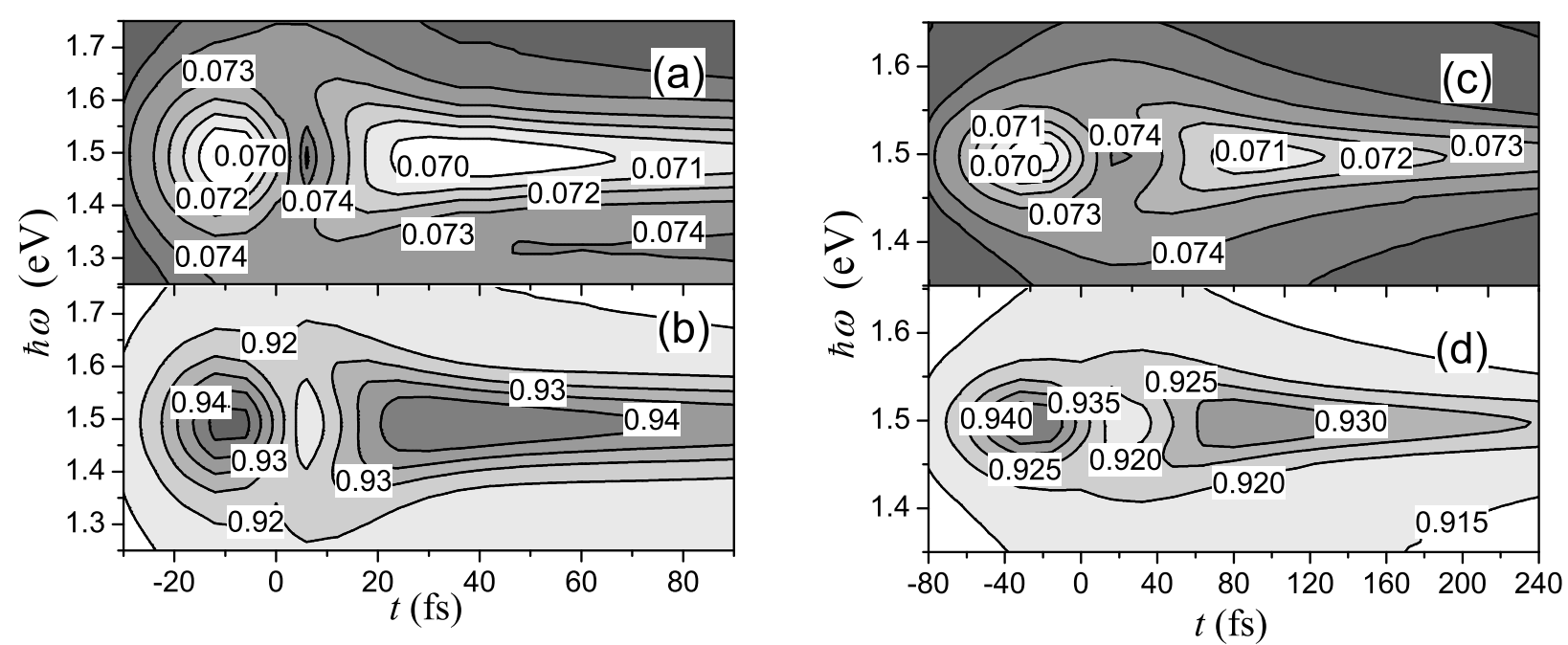

FIG. 5: Contour plots of reflection (a, c) and transmission (b, d) coefficients versus energy of probe radiation $\hbar \omega$ and time for pulse durations $\tau_{p}=30 \mathrm{fs}(\mathrm{a}, \mathrm{b})$ and $80 \mathrm{fs}$ (c, d) at pumping level corresponding to $\mathcal{A}_{\text {ex }}=3 \pi$.

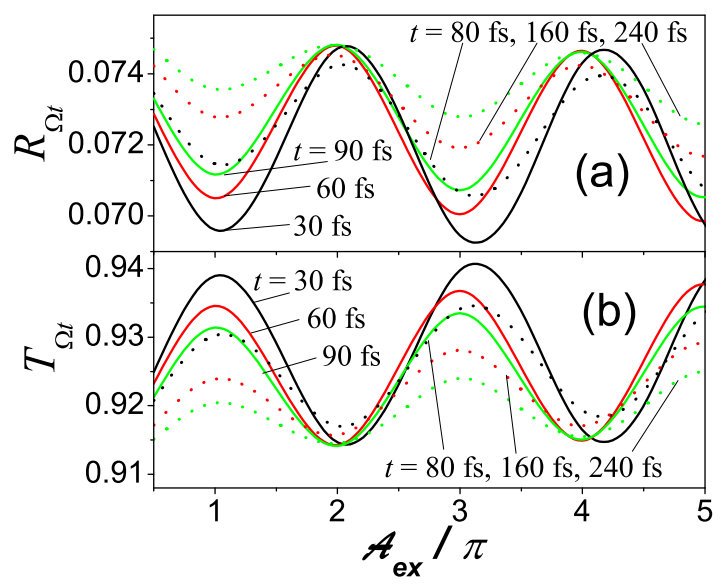

FIG. 6: (Color online) Reflection (a) and transmission (b) versus intensity for $\tau_{p}=30$ fs and 80 fs (solid and dotted curves) at different delay times $t=\tau_{p}, 2 \tau_{p}$, and $3 \tau_{p}$ (marked) and for $\omega=\Omega$.

pumping intensity. A visible damping of this modulation takes place due to the cascade emission of optical phonons, at $t \geq \nu_{p_{\Omega}}^{-1}$.

Our calculations are based on the following assumptions. Since the Coulomb renormalization of interband transitions is weak, [17] we have used a single-particle approach. All homogeneous dephasing mechanisms, including optical phonon emission and carrier-carrier scattering, have been described phenomenologically, through the dephasing time $\tau_{d}$ in Eq.(2). An inhomogeneous broadening due to long-scale disorder is not taken into account, so that the results are valid for a high-quality graphene. The model collision integral (3), which is written through an effective phonon energy $\hbar \omega_{0}$ and a relaxation frequency $\nu_{p}$, is used because the only first step of cascade emission of phonons is essential during the Rabi oscillation process. A detailed calculations can improve a precision of $v_{0}$ used in Eq. (3) and will give a widened peak of distribution at $p_{\Omega}-p_{0}$. The above-listed simplifications of the damping processes do not change the temporal dynamics under consideration and we have demonstrated that the Rabi oscillations are observable in a typical graphene sample. The rest of assumptions [the geometry of normal propagation of radiation, the Gaussian shape of excitation, and the collisionless approximation used in Eqs. (6)] are rather standard.

Summarizing, we have described the mechanisms of coherent nonlinear response of an intrinsic graphene under ultrafast interband excitation. The results obtained demonstrate that the Rabi oscillations, both versus time and versus pumping intensity, can be easily observed for femtosecond time scales (up to $0.1-0.2 \mathrm{ps}$ ) at pumping intensities 
$\sim 3-30 \mathrm{GW} / \mathrm{cm}^{2}$ (which are correspondent to pulse energies $\sim 10-100 \mathrm{~nJ}$ for the sport area $\sim 10^{-4} \mathrm{~cm}^{2}$ )

[1] L. Allen and J.H. Eberly, Optical resonance and two-level atoms (Dover, N. Y. 1987); M. O. Scully and M. S. Zubairy, Quantum Optics (Cambridge University Press, Cambridge 1997).

[2] H. Giessen, A. Knorr, S. Haas, S. W. Koch, S. Linden, J. Kuhl, M. Hetterich, M. Grun, and C. Klingshirn, Phys. Rev. Lett. 81, 4260 (1998); C. Furst, A. Leitenstorfer, A. Nutsch, G. Trankle, and A. Zrenner, Phys. Status Solidi (b) 204, 20 (1997).

[3] A. Schulzgen, R. Binder, M. E. Donovan, M. Lindberg, K. Wundke, H. M. Gibbs, G. Khitrova, and N. Peyghambarian, Phys. Rev. Lett. 82, 2346 (1999); S. T. Cundiff, A. Knorr, J. Feldmann, S. W. Koch, E. O. Gobel, and H. Nickel, Phys. Rev. Lett., 73, 1178 (1994).

[4] F. Rossi and T. Kuhn, Rev. of Mod. Phys., 74, 895 (2002).

[5] V. M. Axt and T. Kuhn, Rep. Prog. Phys. 67, 433 (2004).

[6] J. M. Dawlaty, S. Shivaraman, M. Chandrashekhar, F. Rana, and M. G. Spencer Appl. Phys. Lett. 92, 042116 (2008); D. Sun, Z.-K. Wu, C. Divin, X. Li, C. Berger, W. A. de Heer, P. N.First, and T. B. Norris, Phys. Rev. Lett. 101, 157402 (2008).

[7] R. W. Newson, J. Dean, B. Schmidt, and H. M. van Driel, Opt. Exp. 17, 2326 (2009).

[8] E.M. Lifshitz, L.P. Pitaevskii, and V.B. Berestetskii, Quantum Electrodynamics, (Butterworth-Heinemann, Oxford 1982); P.R. Wallace, Phys. Rev. 71, 622 (1947).

[9] F.T. Vasko and O.E. Raichev, Quantum Kinetic Theory and Applications (Springer, N.Y., 2005).

[10] P. N. Romanets and F.T. Vasko, Phys. Rev. B 81, 085421 (2010).

[11] H. Wang, J. H. Strait, P. A. George, S. Shivaraman, V. B. Shields, Mvs Chandrashekhar, J. Hwang, F. Rana, M. G. Spencer, C. S. Ruiz-Vargas, and J. Park, arXiv:0909.4912.

[12] H. Suzuura and T. Ando, J. Phys. Soc. Japan, 77, 044703 (2008); F. Rana, P. A. George, J. H. Strait, J. Dawlaty, S. Shivaraman, Mvs Chandrashekhar, and M. G. Spencer, Phys. Rev. B 79, 115447 (2009).

[13] D. Potter, Computational Physics (J. Wiley, London, 1973).

[14] The form-factor $w_{t}$ is normalized as $\int_{-\infty}^{\infty} d t w_{t}^{2}=\tau_{p}$. The shape of $w_{t}$ has little effect on the photoexcitation process because the response is mainly dependent on $\tau_{p}$.

[15] L.A. Falkovsky, Phys. Usp. 51887 (2008); T. Stauber, N.M.R. Peres, and A.K. Geim, Phys. Rev. B78, 085432 (2008).

[16] M. V. Strikha and F.T. Vasko, Phys. Rev. B 81, 115413 (2010); M. Bruna and S. Borini, Appl. Phys. Lett. 94, 031901 (2009).

[17] D. E. Sheehy, J. Schmalian, Phys. Rev. B 80, 193411 (2009); L. Yang, J. Deslippe, C.-H. Park, M. L. Cohen, and S. G. Louie, Phys. Rev. Lett. 103, 186802 (2009). 